\title{
LOS PAISAJES CULTURALES PATRIMONIO MUNDIAL COMO HERRAMIENTAS DE GESTIÓN TERRITORIAL. EL CASO DE LA SERRA DE TRAMUNTANA DE MALLORCA
}

\author{
Jaume Mateu i Lladó 1 \\ Departament de Ciències de la Terra. Universitat de les Illes Balears. \\ jaume.mateu.llado@gmail.com
}

\section{RESUMEN}

El concepto de paisaje cultural de la Convención de Patrimonio Mundial se fundamenta en una visión patrimonial del territorio que puede ser una buena herramienta de gestión del paisaje. El uso de los métodos de catalogación y ordenación del Convenio Europeo del Paisaje sobre los paisajes culturales permite aprovechar todo el potencial de ambos convenios en beneficio de la protección del territorio, especialmente a través de las herramientas de participación ciutadana. El presente artículo analiza el caso de la Serra de Tramuntana de Mallorca, designada Patrimonio Mundial en 2011.

Palabras clave: política de paisaje, paisaje cultural, Patrimonio Mundial UNESCO, Convenio Europeo del Paisaje, participación ciudadana, Serra de Tramuntana.

\section{ABSTRACT}

The concept of cultural landscape as it is included in the World Heritage Convention is based in a heritage view that can be useful as a landscape management tool. Moreover, the European Landscape Convention establishes several measures on landscape policy that

Fecha de recepción: noviembre 2012.

Fecha de aceptación: octubre 2013.

1 Este artículo forma parte del Trabajo de Fin de Máster realizado por el autor en el Máster de Análisis, Gestión y Planificación en Áreas Litorales de la Universitat de les Illes Balears, dirigido por la Dra. Joana Maria Seguí Pons (Departament de Ciències de la Terra, Universitat de les Illes Balears). A su vez, es el resultado de los trabajos realizados para el desarrollo de la candidatura de la Serra de Tramuntana a Patrimonio Mundial, de cuyo expediente el autor fue coordinador, bajo la dirección de la Dra. Maria Lluïsa Dubon. 
can be used in a World Heritage Cultural Landscape to improve landscape conservation and protection, specifically through public participation and social governance actions. The article describes the case of the Serra de Tramuntana in Mallorca, inscribed in the World Heritage List in 2011.

Key words: landscape policy, cultural landscape, UNESCO World Heritage, European Landscape Convention, public participation, Serra de Tramuntana.

\section{INTRODUCCIÓN}

La inclusión de la Serra de Tramuntana de Mallorca en la lista de Patrimonio Mundial, en el mes de junio de 2011, ofrece un nuevo enfoque en cuanto a la política de protección y gestión de la comarca. Su consideración como Paisaje Cultural de valor universal por parte del Comité de Patrimonio Mundial de UNESCO supone reconocer el valor patrimonial de la intervención antrópica sobre este territorio, al margen de los incuestionables valores naturales que han fundamentado su protección bajo diferentes figuras.

De los 85 paisajes culturales incluidos hasta el año 2011 en la Lista de Patrimonio Mun$\mathrm{dial}^{2}$, únicamente tres se encontraban en España: los jardines de Aranjuez, el Monte Perdido y la Serra de Tramuntana. En el caso de la Serra de Tramuntana, cabe señalar el hecho de que se trata de un paisaje agrario en su conjunto, de escala comarcal, con una extensión considerable (30.745 hectáreas en la zona núcleo) y de características similares, por su tamaño y rasgos paisajísticos, a otros espacios agrarios del entorno considerados Patrimonio Mundial (los paisajes de Cinque Terre o Amalfi en Italia; el valle de Wachau en Austria; o el paisaje del Alto Douro en Portugal).

En pleno debate sobre el papel de los paisajes culturales y a la consideración del valor patrimonial del paisaje (Silva, 2009) parece oportuno plantear la cuestión de la idoneidad de los paisajes culturales en la Lista de Patrimonio Mundial como herramientas de gestión y protección del territorio. El presente artículo pretende, así, ofrecer un estado de la cuestión de los paisajes culturales y su concepción como herramientas de gestión a partir del caso de la Serra de Tramuntana.

\section{LOS PAISAJES CULTURALES, PAISAJE Y PATRIMONIO}

\section{La definición de los paisajes culturales}

Más allá de su institucionalización en el marco de las políticas definidas en el Convenio Europeo del Paisaje (Consejo de Europa, 2000), el concepto de paisaje es, aún hoy, objeto de múltiples visiones y significados, con una dimensión poliédrica que abarca desde concepciones puramente morfológicas, a visiones estéticas, simbólicas y espirituales (Silva, 2009:311; Cosgrove, 2002). El concepto de paisaje cultural, específicamente, es también objeto de visiones dispares que determinan enfoques quizás complementarios que, sin embargo, parten desde puntos de vista distintos.

\footnotetext{
2 Según la información sobre la Lista de Patrimonio Mundial disponible online en http://whc.unesco.org
} 
Podríamos resumir estas visiones en dos grandes líneas: la visión más morfológica, derivada de la tradición marcada por Carl Sauer y sus discípulos de la escuela de Berkeley; y la visión simbólica, desarrollada en mayor medida por la geografía cultural y humanística anglosajona desde los años 70, a través de autores como Denis Cosgrove o Stephen Daniels (Robertson y Richards, 2003).

La definición morfológica de los paisajes culturales tal y como fue desarrollada por Carl Sauer (Sauer, 1925) entiende como paisaje cultural el resultado de la acción de un grupo social sobre un paisaje natural original, a través de un agente, la cultura, y cuyo resultado sería un paisaje cultural. Se diferencian así dos grandes tipos de paisajes, los naturales (natural landscapes) y los culturales (cultural landscapes). La visión morfológica de los paisajes culturales está muy relacionada con la tradición historicista de la Geografía, de la que adopta un enfoque regionalista en sus métodos de trabajo, basados en el trabajo de campo, el estudio de la evolución histórica del territorio y la lectura del espacio como resultado de la intervención de las diferentes culturas que por él han pasado, y cuyos rastros son descifrables a través del propio paisaje. Tal y como apunta Joaquín Sabaté «resulta más estimulante, en la línea que Sauer inicia, entender el paisaje cultural como un registro del ser humano sobre el territorio; como un texto que se puede interpretar; entendiendo el territorio como construcción humana» (Sabaté, 2008).

La visión simbólica de los paisajes culturales parte fundamentalmente del hecho subjetivo de la contemplación del territorio que es el paisaje. Joan Nogué afirma que «entendiendo [...] el paisaje como una mirada, como una manera de ver y de interpretar, es fácil asumir que las miradas acostumbran a no ser gratuitas, sino que son construidas y responden a una ideología que busca transmitir una determinada forma de apropiación del espacio» (Nogué, 2007:12). El punto de vista simbólico en el paisaje supone entender este paisaje desde el individuo; se entiende que todo paisaje, sea material o imaginado, es una representación de la realidad. El paisaje, al fin y al cabo, es una construcción social, un producto cultural.

Más aún, insiste Joan Nogué: «el paisaje es también un reflejo del poder y una herramienta para establecer, manipular y legitimar las relaciones sociales» (Nogué, op. cit). El método simbólico trata de investigar bajo la capa material de los paisajes para conseguir revelar las relaciones de poder, simbólicas e identitarias que en ellos se esconden.

Por tanto, al no poder establecer una desconexión entre lo material y lo subjetivo, el paisaje cultural se convierte en un reflejo de la forma de ver el territorio por parte de cada grupo social, distinto según el observador. Los paisajes son «invenciones culturales que podemos fechar y analizar» (Roger, 2007); «un paisaje no es jamás reducible a su realidad física (definida como espacio geográfico, ambiente, ecosistema, geosistema o país) sino que la transformación de un país en paisaje supone siempre una metamorfosis, una metafísica, entendida en el sentido dinámico y producida por un complejo proceso que camina desde su misma construcción material a su mera percepción y a su posible simbolización.» (García, et al., 2007; Roger, 1997).

La postmodernidad introduce en los paisajes culturales vistos desde la actual geografía cultural nuevas dimensiones. El triunfo de las imágenes y lo virtual sobre la realidad, los cambios espaciales y temporales que suponen las nuevas tecnologías y la lucha por la singularización y contra la banalización del territorio introducen nuevos matices a la definición de paisaje cultural, «por la emergencia del multiculturalismo, el mestizaje y la hibridez; por 
el triunfo de la imagen, el simulacro, la representación, la realidad virtual» (Nogué, 2008). Así pues, el análisis de los paisajes culturales desde el punto de vista simbólico debe permitir descrifrar, además de sus elementos materiales, sus valores culturales de carácter inmaterial que reflejan también un modo específico de relacionarse con la naturaleza y con el espacio por parte de la sociedad.

La concepción del paisaje como patrimonio es quizás uno de los puentes entre las dos visiones de los paisajes culturales: ante los procesos de banalización, pérdida de carácter o virtualización del territorio que acarrea su evolución en el marco de la globalización, los paisajes culturales aparecen como refugios de la identidad, como singularidades de gran belleza y significado histórico, como espacios con memoria con los que la sociedad busca recuperar la relación con un territorio cada vez más homogéneo y aterritorial.

\section{Los paisajes culturales en la Lista de Patrimonio Mundial de UNESCO}

La primera institución internacional en reconocer el valor patrimonial de los paisajes culturales a través de un instrumento jurídico y legal fue el Comité de Patrimonio Mundial de la UNESCO. Después de algunos años de debate sobre la necesidad de incluir nuevas formas de patrimonio en la Lista de Patrimonio Mundial (Fowler, 2002) como los sistemas agrícolas tradicionales, o las «obras combinadas entre hombre y naturaleza» establecidas en la Convención de Patrimonio Mundial de 1972, en el año 1992 se consideran, por primera vez, los paisajes culturales como elementos susceptibles de formar parte de la lista de Patrimonio Mundial (Von Droste et al, 1999).

Los paisajes culturales, tal y como los define UNESCO, se conciben básicamente a partir de la visión saueriana del término:

«Los paisajes culturales son bienes culturales y representan las «obras conjuntas del hombre y la naturaleza» mencionadas en el Artículo 1 de la Convención. Ilustran la evolución de la sociedad y los asentamientos humanos a lo largo de los años, bajo la influencia de las limitaciones y/o de las ventajas que presenta el entorno natural y de fuerzas sociales, económicas y culturales sucesivas, internas y externas» (UNESCO, 2006).

A partir de aquí, el Comité de Patrimonio Mundial adopta la división de los paisajes culturales en tres grandes categorías:

1) Paisajes claramente definidos, concebidos y creados intencionalmente. Comprenden los jardines y parques diseñados por razones estéticas (Versalles).

2) Paisajes evolucionados orgánicamente, es decir, fruto de la acción de un grupo cultural sobre el medio físico original a lo largo de la historia. Éstos pueden ser paisajes fósiles, cuando la «cultura» que los creó ya ha dejado de existir, o paisajes vivos, en los cuales el proceso evolutivo que los creó sigue existiendo (el paisaje vitivinícola de Saint Émilion, en Burdeos).

3) Paisajes asociativos, enclaves en general naturales a los que se les asocian valores de tipo religioso, artístico o cultural, cuyo significado es más importante que no sus evidencias materiales (el Parque Nacional de Tongariro, en Nueva Zelanda).

En 2011 la lista de Patrimonio Mundial contaba con 85 sitios inscritos como paisajes culturales, un $9 \%$ del total. Es destacable la diversidad de espacios incluidos en esta categoría, desde centros urbanos históricos como los de Puebla (Méjico) o Valletta (Malta), a paisajes 
agrícolas como los de los viñedos (Lavaux, en Suiza; Saint Émilion, en Francia; o Tokaj, en Hungría), el café (Colombia, Etiopía, Cuba) o el arroz (Filipinas); antiguas zonas industriales y mineras como las de Blaenavon en el Reino Unido o Røros en Noruega; jardines botánicos como los de Kew (Reino Unido), o espacios de significación espiritual o religiosa como Ouadi Qadisha en Líbano, el Sukur en Nigeria o Uluru-kata Tjuta en Australia. Prácticamente la mitad (47\%) de los paisajes culturales Patrimonio Mundial se encuentran en Europa y América del Norte (figura 1). El resto se distribuyen de forma similar entre Asia / Pacífico (19\%), América Latina y Caribe (15\%) y África (14\%).

Figura 1

DISTRIBUCIÓN REGIONAL DE LOS PAISAJES CULTURALES PATRIMONIO MUNDIAL (2011)

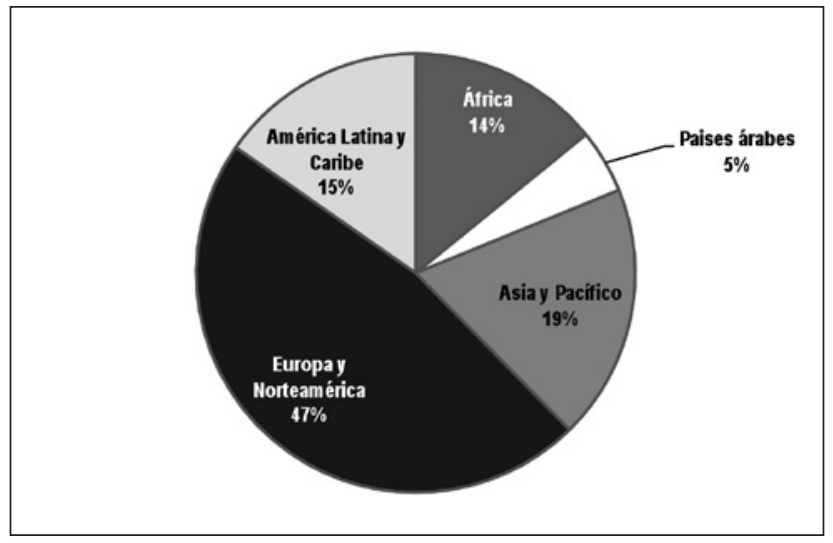

Fuente: UNESCO - Centro de Patrimonio Mundial

La designación de paisajes culturales bajo el paraguas de la lista de Patrimonio Mundial ha permitido abrir el concepto de patrimonio hacia nuevos lugares, hacia nuevos espacios. Este proceso es, al fin y al cabo, una evolución del concepto de Patrimonio Mundial, inicialmente reservado casi exclusivamente a bienes de interés artístico o arqueológico, para abrirse primero hacia parajes naturales y posteriormente hacia los paisajes culturales (Roudié, 2002). En este sentido, la consideración de elementos intangibles del paisaje a través de los «paisajes culturales asociativos» ha supuesto un gran avance en cuanto al reconocimiento de la relación de muchos pueblos indígenas con el territorio. También han sido reconocidos patrimonialmente sistemas de gestión territorial ancestrales y únicos, la diversidad cultural reflejada sobre el espacio, los elementos vinculados a la agricultura, a la gestión del agua o de zonas con recursos naturales escasos, y se han dado también avances significativos en cuanto a la interpretación, divulgación, rehabilitación y desarrollo de muchos territorios (Rössler, 2006).

En paralelo, la complejidad derivada de la gestión de estos espacios y la gran atracción que la marca Patrimonio Mundial ofrece a nivel turístico ha supuesto la aparición de nuevas amenazas y retos. Los excesos en forma de masificación turística con un gran impacto sobre la población local ponen de manifiesto la necesidad de evolucionar hacia modelos de conser- 
vación basados en la sostenibilidad en el uso del territorio, la implicación directa de la población local en su gestión y el apoyo a la conservación de los sistemas agrarios tradicionales. En caso contrario, la inscripción de determinados enclaves en la Lista de Patrimonio Mundial puede conducir, debido a un exceso de presión de los visitantes y a los cambios socioeconómicos derivados de la terciarización, a su abandono y degradación.

\section{EL CASO DE LA SERRA DE TRAMUNTANA DE MALLORCA}

\section{Descripción de la comarca}

La Serra de Tramuntana es la principal región montañosa de la isla de Mallorca. Con una extensión cercana a las 90.000 hectáreas, se trata de una cordillera calcárea con cimas que llegan a los 1.400 metros de altitud y con diferencias orográficas entre su vertiente litoral, escarpada y abrupta, y su vertiente suroriental que cae más suavemente sobre la zona interior de la isla (figura 2). Esta peculiaridad hace de la Serra de Tramuntana una isla dentro de otra isla, un espacio de evolución diferenciada respecto al resto del territorio, y con unos valores simbólicos e icónicos muy importantes para la población mallorquina (Pujol, 2000).

Figura 2

UBICACIÓN DE LA SERRA DE TRAMUNTANA

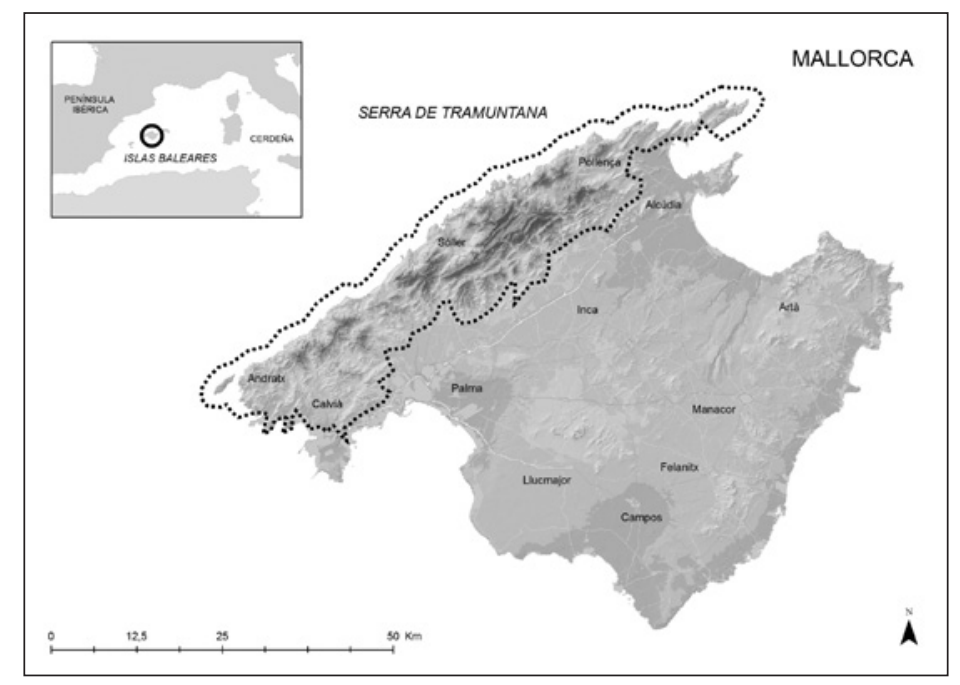

Fuente: Elaboración propia a partir de datos de SITIBSA.

La riqueza biogeográfica de la Serra de Tramuntana, jalonada por la presencia de la mayor parte de especies endémicas, raras o relictuales de las Baleares (RAMON, 2002) se basa en sus peculiaridades ecológicas, geomorfológicas y climáticas. La Serra acoge espacios de gran valor ecológico, como lo demuestra el hecho de concentrar gran parte de las zonas incluidas en la Red Natura 2000 de las Baleares. 
A su vez, el territorio de la Serra de Tramuntana es también un espacio fuertemente antropizado. La presión demográfica de la isla de Mallorca en diferentes momentos históricos ha conllevado la necesidad de aprovechar al máximo los recursos disponibles, lo que en la Serra de Tramuntana se traduce en el abancalamiento del territorio para la obtención de espacios de cultivo y el uso de sistemas tradicionales para la captación de los recursos hídricos, favorecidos por la presencia de mananatiales cársticos y la mayor pluviometría de la zona central (GRIMALT et. al., 2002). La orografía también le supone una evolución diferente del resto de la isla en cuanto a la transformación turística del territorio. Exceptuando las zonas periféricas de Andratx, Calvià, Pollença y el Port de Sóller, en las que el desarrollo turístico de masas, a partir de los años 60, ha sido intenso, el resto de municipios han sufrido una menor transformación turística de su zona litoral. Por contra, cabe destacar en ellos procesos de terciarización del espacio, abandono rural, y uso turístico a través de hoteles de interior, rurales y otras modalidades turísticas en medio rural y natural (Salvá, 1998; Binimelis et al, 1999). La lógica espacial en el contexto europeo e internacional actual acarrea una mayor presión turística y residencial. Se produce así un gran aumento de la presión inmobiliaria y la orientación casi exclusiva del sistema económico hacia el sector terciario, especialmente turístico e inmobiliario. Como consecuencia se da un abandono progresivo de los sistemas tradicionales de cultivo, lo que conlleva la pérdida de valores culturales, etnológicos y patrimoniales con la consecuente degradación paisajística, aún cuando buena parte del territorio esté protegido por la legislación urbanística y medioambiental.

En cualquier caso, los valores de la Serra de Tramuntana han sido reconocidos a través de diversas figuras legislativas en el marco de la protección de espacios naturales y protección del patrimonio histórico y artístico (tabla 1).

Tabla 1

FIGURAS DE PROTECCIÓN DE LA SERRADE TRAMUNTANA

\begin{tabular}{|l|c|}
\hline \multicolumn{1}{|c|}{ FIGURA DE PROTECCIÓN } & AÑO \\
\hline Paraje Pintoresco & 1972 \\
\hline $\begin{array}{l}\text { Ley 1/1991, de espacios naturales y régimen urbanístico de las áreas de especial } \\
\text { protección de las Baleares (LEN) }\end{array}$ & 1991 \\
\hline $\begin{array}{l}\text { Ley autonómica 5/2005, de 26 de mayo, para la conservación de los espacios } \\
\text { de relevancia ambiental (LECO), y, en base a esta ley, el Decreto 19/2007 } \\
\text { de aprobación del Plan de Ordenación de Recursos Naturales de la Serra de } \\
\text { Tramuntana. }\end{array}$ & $2005 / 2007$ \\
\hline $\begin{array}{l}\text { Decreto 28/2006, de declaración de Zonas de Especial Protección para las Aves } \\
\text { (ZEPA) en el ámbito de las Illes Balears, y Decreto 29/2006, de ampliación de } \\
\text { la lista de Lugares de Importancia Comunitaria (LIC) y se declaran Zonas de } \\
\text { Especial Protección para las Aves. }\end{array}$ & 2006 \\
\hline
\end{tabular}

Fuente: Elaboración propia. 


\section{La Serra de Tramuntana como paisaje cultural}

\subsection{El proceso de inscripción en la Lista de Patrimonio Mundial.}

La historia de los procesos de protección de la Serra de Tramuntana está marcada por diferentes intentos de establecer un marco de protección adecuado para sus características y valores. Desde la primera propuesta de declarar las propiedades históricas del Archiduque Luís Salvador de Austria como Parque Nacional, en 1923, (Cañellas, 1997), se han sucedido diferentes acciones de protección y ordenación del territorio, algunas fallidas como las de su ordenación mediante Plan Territorial Parcial (AAVV, 1988) o la su intento de declaración como Parque Natural en base a la legislación estatal en el año 2002. Diferentes instrumentos, de planificación territorial, urbanísica, turística, de desarrollo rural, de conservación de flora y fauna, de conservación del patrimonio, contemplan acciones específicas para la Serra de Tramuntana, a nivel local, estatal e internacional. En todos ellos aparece uno de los condicionantes a los que ha cabido enfrentarse a la hora de plantear las figuras administrativas de protección del territorio de la Serra de Tramuntana: la elevada antropización de su territorio. Los usos tradicionales del territorio constituyen una de las piezas clave para mantener, a la vez, los valores naturales.

La propuesta de inscripción de la Serra de Tramuntana de Mallorca en la lista de Patrimonio Mundial en la categoría de paisaje cultural fue uno de los objetivos que planteó la Estrategia del Paisaje de Mallorca, desarrollada por el Consell de Mallorca a partir del año 2008 (Dubon, 2009), con el objeto de abordar la cuestión de la protección de la Serra de Tramuntana no solo desde un punto de vista de su conservación, en el sentido de prohibir determinadas actividades que suponen un grave impacto, principalmente urbanizaciones; sino ofrecer, de alguna manera, estímulos a aquellas actividades que se revelan como necesarias para el mantenimiento de su peculiar paisaje, planteando una gestión en positivo paralela a las figuras de conservación y protección ya existentes.

La Estrategia del Paisaje de Mallorca es fundamentalmente una propuesta de aplicación de la Convención Europea del Paisaje a escala local utilizando las competencias propias del Consell de Mallorca en materia de ordenación del territorio, urbanismo y patrimonio histórico, así como a las políticas en materia de turismo, medio ambiente y desarrollo local llevadas a cabo por dicha institución en el marco de sus atribuciones básicas, que incluían a su vez, herramientas de participación ciudadana y gobernanza.

Así, la candidatura de la Serra de Tramuntana a Patrimonio Mundial fue concebida para cumplir con algunos de los objetivos incluidos en la propia Convención Europea del Paisaje: dar a conocer y calificar los paisajes para educar, sensibilizar y actuar; definir y aplicar una política específica de paisaje para la protección, gestión y ordenación de los paisajes; y desarrollar y aplicar procedimientos de participación pública. Desde este punto de vista la propuesta trata de hacer converger, en un proyecto único, los valores y propuestas derivados de la Convención Europea del Paisaje y los de la Convención de Patrimonio Mundial de 1972, aun asumiendo las diferentes visiones de paisaje que contienen ambos documentos ${ }^{3}$.

3 Las diferentes visiones de paisaje del Centro de Patrimonio Mundial y el Convenio Europeo del Paisaje aparecen reflejadas, entre otros, en un texto de Peter Fowler sobre el estado de la cuestión de los paisajes culturales Patrimonio Mundial entre 1992 y 2002 (FOWLER, 2002:17). 
Los métodos de gobernanza y participación social en la definicion de propuestas de conservación y gestión de paisaje aplicadas a la propuesta de inclusión en la lista de Patrimonio Mundial se inspiran en las definiciones del Convenio Europeo del Paisaje. Se propone a su vez aprovechar el valor simbólico, de orgullo local y de proyecto territorial común que puede suponer el proceso de designación de un elemento como Patrimonio Mundial para establecer políticas de sensibilización y concienciación social en la protección y defensa del territorio, y que a su vez permitan recuperar, de algún modo, las actividades agrarias tradicionales que sustentan el paisaje.

El proceso de designación de la Serra de Tramuntana como Patrimonio Mundial se fundamenta así en tres grandes elementos:

En primer lugar, la identificación y delimitación de los valores paisajísticos del territorio, y su documentación a través de toda la información disponible, para completar el expediente de designación como paisaje cultural Patrimonio Mundial ante UNESCO, siguiendo sus estrictas recomendaciones (UNESCO, 2006). Esta identificación de valores del paisaje es especialmente relevante para justificar el llamado «Valor Universal Excepcional», así como cumplir con alguno de los diez criterios en los que debe basarse la inscripción de un bien en la Lista de Patrimonio Mundial.

En segundo lugar, la elaboración e implementación de un Plan de Gestión, utilizando un proceso de participación ciudadana en la diagnosis y propuesta de acciones de gestión con el fin de implicar a todos los agentes sociales de la Serra de Tramuntana en la gestión del paisaje.

Y finalmente, el desarrollo de campañas de apoyo a la candidatura como herramienta de sensibilización y concienciación ciudadana en torno al paisaje.

El proceso de designación de la Serra de Tramuntana en la Lista de Patrimonio Mundial (tabla 2) se llevó a cabo básicamente entre agosto de 2008, cuando se dio inicio a la elaboración del expediente, y junio de 2011, cuando el Comité de Patrimonio Mundial acordó su inclusión en la Lista de Patrimonio Mundial en la 35a sesión celebrada en París ${ }^{4}$.

Tabla 2

PROCESO DE INSCRIPCIÓN DE LA SERRA DE TRAMUNTANA EN LA LISTA DE PATRIMONIO MUNDIAL

\begin{tabular}{|l|l|}
\hline 1996 & Inclusión de la Serra de Tramuntana en la Lista Indicativa de Patrimonio Mundial \\
\hline Agosto 2008 & Inicio expediente por parte del Consell de Mallorca \\
\hline Julio 2009 & $\begin{array}{l}\text { Elección de la Serra de Tramuntana como candidata española a Patrimonio } \\
\text { Mundial (Consejo Nacional de Patrimonio) }\end{array}$ \\
\hline Septiembre 2009 & Remisión borrador expediente al Centro de Patrimonio Mundial de París \\
\hline Enero 2010 & Remisión expediente completo al Centro de Patrimonio Mundial de París \\
\hline Octubre 2010 & Misión de evaluación sobre el terreno \\
\hline Junio 2011 & Inscripción de la Serra de Tramuntana en la Lista de Patrimonio Mundial \\
\hline
\end{tabular}

Fuente: Elaboración propia.

4 Decisión 35COM 8B.34 del Comité de Patrimonio Mundial (http://whc.unesco.org/archive/2011/whc1135com-20e.pdf, consultada en septiembre 2012) 


\subsection{Los valores paisajísticos de la Serra de Tramuntana y su delimitación}

El punto de partida para la consideración de la Serra de Tramuntana como paisaje cultural es su encaje en las definicion de paisajes culturales de UNESCO como obra conjunta entre hombre y naturaleza que ha evolucionado orgánicamente como respuesta antrópica a la necesidad de aprovechar los recursos que ofrece el medio físico original para facilitar la ocupación humana del espacio. En el caso de la Serra de Tramuntana se considera además que es un paisaje vivo, en el sentido que la cultura de la que provienen los conocimientos técnicos y la estructura social para configurar dicho paisaje en cierto modo se mantiene.

El paisaje cultural de la Serra de Tramuntana se construye históricamente a partir de dos elementos principales: el uso de la técnica de la piedra en seco para obtener nuevos espacios de cultivo (Grimalt y Blázquez, 1989; Reynés, 2000), y los sistemas de captación, transporte y almacenamiento de agua que permiten aprovechar las fuentes y manantiales naturales y establecer zonas de regadío en un contexto de escasez hídrica mediterránea. El uso intensivo de la piedra en seco para modelar el territorio, junto con el control del agua, ha permitido en la Serra sostener las grandes fincas rurales (las possessions), que actúan como unidades de producción agraria, y constituyen las teselas del mosaico paisajístico. Es también la distribución de los sistemas hidráulicos la que por lo general explica la ubicación y configuración de los diferentes asentamientos. Una extensa red de caminos y senderos ha permitido conectar fincas y pueblos, así como zonas de explotación forestal, cultivos, o elementos de significación religiosa (ermitas, santuarios o iglesias).

A su vez, las características orográficas del terreno, así como su ubicación costera, aportan valores escénicos y estéticos muy relevantes a este paisaje, inspiradores de numerosas obras literarias, musicales y pictóricas.

\section{a) Evolución histórica del territorio en la Serra de Tramuntana}

La evolución histórica del territorio es una de las claves para comprender la actual configuración del paisaje de la Serra de Tramuntana, y justificar el valor patrimonial o cultural de sus elementos. En este sentido, las huellas del paisaje cultural más relevantes de la Serra se basan en los sucesivos hitos históricos acacidos desde el siglo X: la ocupación islámica de la isla (ss. X-XIII), la época feudal y renacentista (ss. XIII - XVIII); y la época moderna, a partir del siglo XIX. Los efectos de la postmodernidad sobre el territorio deben considerarse asociados a la entrada en la escala global a través del turismo.

Mallorca es la piedra con la que tropiezan cada una de las civilizaciones que pretenden controlar estratégicamente el Mediterráneo. La cultura islámica, en su influencia hacia el norte. La cultura cristiana/europea, en su influencia hacia el sur. A partir del siglo XVIII, la entrada en la época moderna fundamentada en la pérdida de valor geopolítico del Mediterráneo y la incipiente industrialización. Finalmente, las diferentes fases evolutivas del turismo (Gormsen, 1997) que conducen a los procesos de compresión espacio-temporal en Mallorca (Bauzà, 2009), cambian el modo de relación con el territorio inmediato en favor de la escala global. Todo ello en un marco territorial marcado por la insularidad y la dependencia con el exterior, que condiciona cultivos, necesidades y estrategias de explotación de los recursos a diferentes escalas en función de la tecnología o de los conocimientos disponibles en cada época. 
Por tanto, la configuración del actual paisaje rural en Mallorca tiene su raiz en la ocupación islámica de la isla, entre los años 903 y 1229. Entre la la época romana (123 aC - 454 dC) y el año 903 se produce un periodo de transición que permite que los nuevos pobladores islámicos establezcan su modelo de desarrollo territorial prácticamente sin condicionantes previos (Rullán, 2004:88). El modelo islámico de ocupación se fundamenta, pues, en la división clánica o tribal del territorio y en la dispersión de la población a través de pequeños asentamientos rurales, las alqueries (al-Garya) y los rafals (rahal), ubicados generalmente en torno a un sistema hidráulico sobre el que se desarrolla una agricultura de regadío que permite el autoabastecimiento y el comercio a pequeña escala (Kirchner, 1994). El pago de impuestos en moneda facilitaba una cierta independencia en la elección de los productos a cultivar y con los que comerciar. A la vez, el dominio de las técnicas de captación y distribución de agua en entornos áridos que tenían los pobladores islámicos les permitió diseñar una red de espacios irrigados muy notable (Barceló et. al., 1986) en torno a la cual desarrollaron diferentes asentamientos, sistemas de drenaje y espacios cultivables generalmente comunitarios y regulados mediante los llamados ma'jil, una organización social basada en la regulación de la distribución del agua (Carbonero, 1984). El territorio no irrigado se dedicaba a cultivos de secano como el olivo, en las zonas cercanas a los nucleos de población, y a pastos y bosque el resto del territorio.

En la Serra de Tramuntana, las características pluviométricas y la profusión de manantiales de origen natural permitieron desarrollar una gran parte de los sistemas hidráulicos islámicos, de los que son buenos ejemplos los valles de la zona de piedemonte (Santa Maria, Esporles, Bunyola, Alaró) (Riera y Soberats, 1992). El paisaje rural de la época se basaba, pues, en una dispersión de pequeñas zonas de regadío en torno a una matriz boscosa que se dedicaba a pastos y aprovechamiento forestal.

La conquista cristiana de 1229 provocará una transición del sistema islámico al sistema feudal de ocupación. La ocupación catalana de la isla de Mallorca supone el reparto de todo el territorio entre los diferentes señores feudales que participaron en la contienda. Sobre la base de la ubicación de las antiguas alquerías, los nobles catalanes se reparten el territorio configurando una estructura de grandes propiedades y convirtiendo a los habitantes en vasallos feudales, obligados a pagar una renta anual en especies. Se rompe así el modelo agrícola musulmán, eficiente, diversificado y comunal, y se impone la expansión del cultivo del trigo, de la vid y del olivo (Kirchner y Soto, 2006). En la Serra, un espacio orográficamente dificultoso para el trigo, se produce la expansión del cultivo del olivo, a través de bancales, para la obtención del aceite, destinado a la exportación.

$\mathrm{El}$ «modelo territorial» que surge a partir de la conquista cristiana permite el avance, entre los siglos XIV y XVIII, de las zonas cultivadas en detrimento de las áreas boscosas, y su expansión hasta zonas prácticamente incultivables, gracias al uso del abancalamiento y a la necesidad de aprovechar hasta el último rincón del territorio de la isla debido a las fluctuaciones demográficas y a la demanda de trigo y aceite. La época moderna, a partir de los siglos XIX y XX, conllevará la introducción de nuevos cultivos, el crecimiento demográfico y el desarrollo industrial y turístico. La desamortización permite la aparición de zonas de pequeña propiedad cerca de los núcleos urbanos. La expansión del cultivo de la vid y el almendro; la exportación de cítricos y otros productos (sobretodo en el caso de Sóller) a Europa y América; el desarrollo de nuevas infraestructuras viarias y el desarrollo de una 
cierta industria téxtil (Sóller, Esporles) gracias de nuevo al aprovechamiento de los recursos hídricos permiten, a la vez, una nueva fase de desarrollo en la Serra de Tramuntana, que tiene su reflejo en el paisaje.

La puesta en valor del paisaje como recurso turístico se inicia, precisamente, con la descubierta de los paisajes isleños por parte de los viajeros ilustrados centroeuropeos, como fue el caso del Archiduque Luis Salvador de Austria. A partir del siglo XX se impulsa, en la Serra de Tramuntana, la construcción de nuevas infraestructuras de comunicacion, miradores y el desarrollo de una incipiente oferta de alojamiento que sería la base del desarrollo del turismo de masas.

b) La identificación y delimitación de los valores paisajísticos

La evolución histórica del territorio de Tramuntana es la que permite fijar y justificar el llamado «Valor Universal Excepcional» establecido en la Convención de Patirmonio Mundial de 1972. En el caso de la Serra se justifica este valor a partir de tres de los diez criterios marcados por dicha Convención (Dubon, 2010; UNESCO, op. cit.):

- Criterio II: Testimoniar un importante intercambio de valores humanos a lo largo de un periodo de tiempo o dentro de un área cultural del mundo, en el desarrollo de la arquitectura o tecnología, artes monumentales, urbanismo o diseño paisajístico. En la Serra de Tramuntana el intercambio de valores culturales para el desarrollo de un territorio se ejemplifica en la combinación entre la técnica hidráulica de origen islámico, y la expansión del cultivo del olivo y el trigo, de cultura cristiana, de la que proviene el abancalamiento del territorio y la configuración territorial a partir de las grandes fincas, las possessions.

- Criterio IV: Ser un ejemplo eminentemente representativo de un tipo de construcción o de un conjunto arquitectónico o tecnológico, o de paisaje que ilustre uno o varios periodos significativos de la historia humana. El grado de desarrollo alcanzado por las técnicas de piedra en seco en la construcción de terrazas, caminos y muros; y de las técnicas hidráulicas para la captación y uso del agua, basadas en la técnica del qânat, hacen de la Serra de Tramuntana uno de los espacios más representativos del Mediterráneo en cuanto a uso y manejo de técnicas agrícolas tradicionales.

- Criterio (V) Ser un ejemplo destacado de formas tradicionales de asentamiento humano o de utilización de la tierra o del mar, representativas de una cultura (o de varias culturas), o de interacción del hombre con el medio, sobre todo cuando éste se ha vuelto vulnerable debido al impacto provocado por cambios irreversibles. Los patrones de asentamiento en la Serra de Tramuntana representan un buen ejemplo de la adaptación humana a condiciones ambientales poco favorables, utilizando los recursos que ofrece el medio para subsistir. El resultado de esta adaptación es un gradiente altitudinal de usos del suelo caracterizado por las zonas de huerta en los espacios de irrigación; cultivos de vid y olivo desde el nivel del mar hasta el límite altitudinal de cultivo (sobre los $800 \mathrm{~m}$.); encinares para la explotación del bosque y espacios residuales -de alto valor ecológico- en las cumbres y zonas costeras.

La delimitación de la zona propuesta como paisaje cultural Patrimonio Mundial se llevó a cabo siguiendo las recomendaciones del Centro de Patrimonio Mundial, que requieren la 
delimitación de una zona principal, o zona núcleo, y una zona tampón, o zona de transición entre la zona núcleo y el resto del territorio en el que no existen los valores que justifican la propuesta de inscripción. Para ello se construyó un Sistema de Información Geográfica en el cual se incluyeron 12 capas de información sobre los diferentes elementos que configuran su paisaje: áreas abancaladas, sistemas hidráulicos tradicionales, sistemas tradicionales de control de la erosión y excesos hídricos; ubicación de almazaras; ubicación de puntos de recogida de la nieve (neveras); encinares; fincas públicas; red de caminos; yacimientos arqueológicos; elementos de arquitectura defensiva (castillos, torres de defensa, torres de vigía); jardines históricos y casas de «possessió» con valor patrimonial. La rasterización de cada una de estas capas (convirtiendo cada celda en valores de presencia/ausencia del criterio especificado) y su superposición (sumando cada una de las celdas) dio lugar a un mapa de densidad de valores paisajísticos. Sobre este mapa posteriormente se superponieron las delimitaciones de las tres figuras principales de protección de la comarca, como son la delimitación de la zona declarada como «Paisaje Pintoresco»; la delimitación de las Áreas Naturales de Especial Interés de la Serra de Tramuntana, según la definición de la ley balear 1/1991 y las protecciones establecidas en el Decreto 19/2007 por el que se aprueba el Plan de Ordenación de los Recursos Naturales. De este modo se garantizó que toda la zona esté protegida bajo alguna de las figuras legales existentes.

Sobre esta información se trazó el límite de la zona núcleo y la zona tampón, a la que se añadió también una franja de protección costera para garantizar la protección de la zona núcleo por el litoral. La zona núcleo delimitada abarca una extensión de 30.745 hectáreas, mientras que la extensión de la zona tampón es de 52.760 hectáreas en la zona terrestre y 25.857 en el espacio marítimo (figura 3). Sumando ambas zonas se supera la superficie englobada bajo la Ley de Espacios Naturales de 1991 y el límite incluido bajo la figura de Paraje Natural.

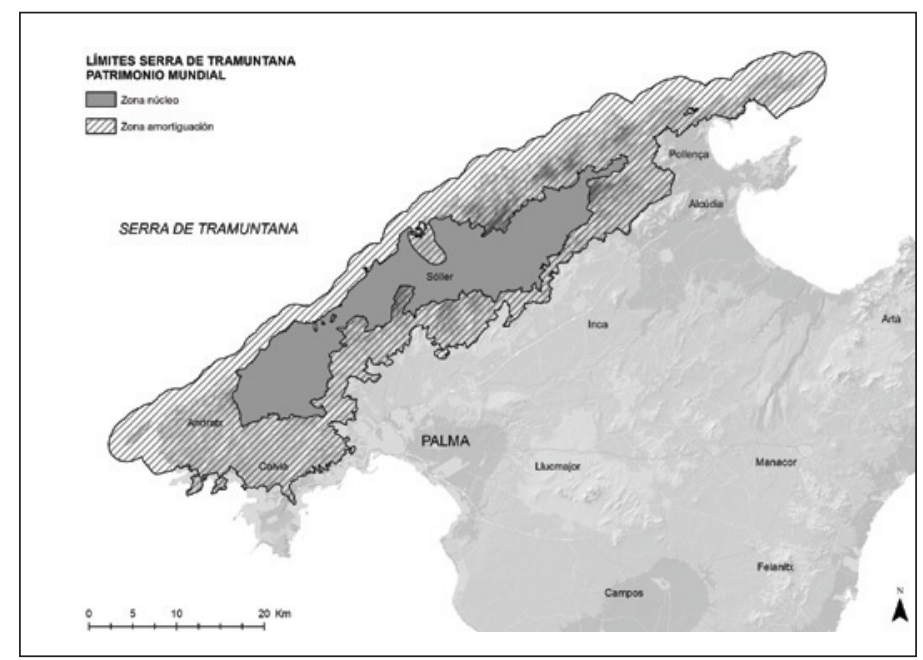

Fuente: Elaboración propia a partir de datos del Consell de Mallorca y SITIBSA. 


\subsection{Desarrollo e implementación del Plan de Gestión}

El Plan de Gestión es un elemento indispensable para cualquier bien que pretenda ser inscrito en la lista de Patrimonio Mundial, que debe demostrar «una adecuada legislación a largo plazo y herramientas de protección de tipo administrativo o tradicional para garantizar la conservación del bien» (UNESCO, op. cit.).

El Plan de Gestión del paisaje cultural de la Serra de Tramuntana se establece para dar respuesta a las amenazas principales detectadas, principalmente en relación al abandono de las actividades tradicionales; la pérdida de autenticidad; la presión recreativa, turística y urbanística; y el peligro de conversión del entorno en un paisaje de «museo» (Dubon, 2010). Para ello, el Plan de Gestión establece como objetivo principal «desarrollar estrategias, objetivos, programas, proyectos y herramientas de gestión para la protección, conservación, divulgación, y desarrollo económico sostenible de los valores del paisaje de la Serra de Tramuntana».

Se trata, por consiguiente, de un plan dirigido a la conservación del paisaje y sus valores, habida cuenta que la Serra de Tramuntana cuenta con mecanismos específicos de protección ambiental y de patrimonio histórico. Para ello, se diseñó una estrategia de coordinación y protección de las actividades sociales y económicas que permitan conservar los valores del paisaje, así como fomentar la sensibilización y la toma de conciencia, por parte de los propios habitantes de la comarca, de los valores culturales y paisajísticos del territorio.

En este sentido, tres son los pilares básicos de este Plan de Gestión:

1) La creación de cuatro programas de acción (tabla 3) que den respuesta al objetivo planteado: desarrollo económico local; divulgación y educación; conservación del patrimonio; gestión de visitantes. Una de las prioridades de los programas es la de mantener la agricultura y la ganadería en la zona, como base para la conservación de toda la estructura agraria que fundamenta el paisaje, fuertemente amenazada debido a su baja competitividad.

2) La creación de una entidad de gestión que actue como coordinadora de las diferentes administraciones presentes en la Serra de Tramuntana, con capacidad de acción en los ámbitos competenciales de ordenación del territorio y urbanismo; patrimonio histórico y cultural; agricultura y desarrollo rural; medio ambiente y biodiversidad; turismo. Este órgano de coordinación interadministrativa se establece con vocación de entidad coordinadora pero con carácter ejecutivo, que debe permitir condicionar todas las políticas sectoriales a los objetivos y programas que determina el Plan de Gestión.

3) El apoyo a la toma de decisiones a través de un proceso de participación ciudadana, que permita priorizar las acciones a desarrollar e involucrar a los principales agentes sociales y económicos de la comarca en la gestión directa del territorio.

El proceso de participación ciudadana se desarrolló, en una primera fase, entre octubre de 2009 y enero de 2010, en paralelo a la elaboración del resto de documentación que conforma el expediente. En ellas tomaron parte 46 representantes de diferentes asociaciones y colectivos: ayuntamientos, administraciones supramunicipales, representantes de los propietarios de fincas, agricultores y gana- 
deros, entidades excursionistas y deportivas, entidades de conservación de la naturaleza y el patrimonio, empresas turísticas, escuelas e institutos, centros de investigación, entre otros. El órgano de gestión del paisaje cultural, a su vez, se creó en mayo de 2011, en forma de consorcio público auspiciado por el Gobierno autonómico y el Consell de Mallorca, que prevé asimismo la incorporación de los 19 ayuntamientos incluidos en la delimitación del paisaje cultural de la Serra de Tramuntana.

Tabla 3

\section{EJES PRINCIPALES DEL PLAN DE GESTIÓN DE LA SERRA DE TRAMUNTANA}

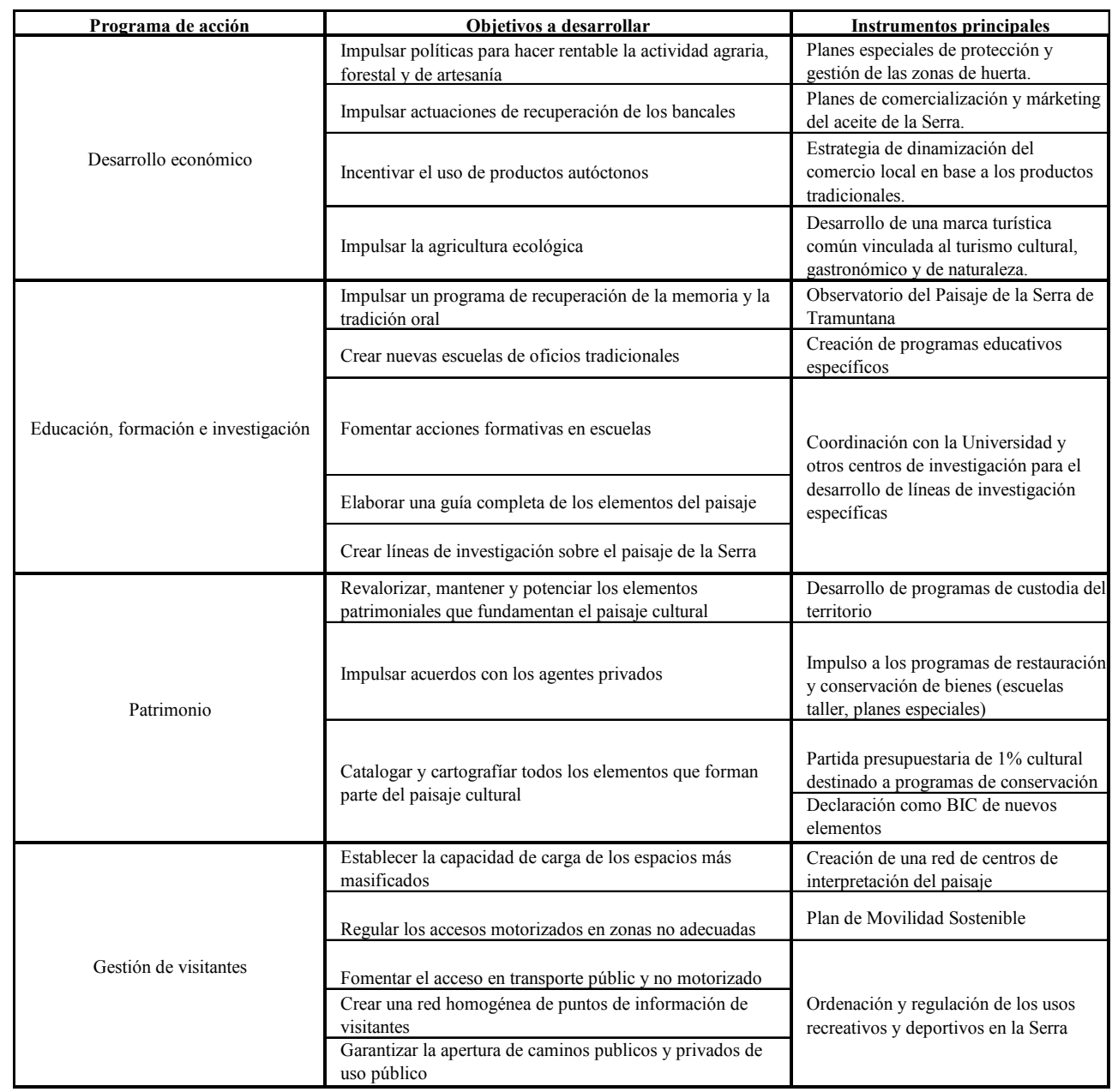

Fuente: Elaboración propia a partir de datos del expediente de declaración de la Serra de Tramuntana (Dubon, 2010) 


\section{DISCUSIÓN Y CONCLUSIONES}

El proceso de declaración de la Serra de Tramuntana como paisaje cultural Patrimonio Mundial ha servido para dar un nuevo impulso a la estrategia de conservación de la comarca, basado en un cambio de enfoque de las políticas de conservación. La Serra de Tramuntana cuenta con diversas figuras de protección que, si bien han permitido proteger el territorio de las amenazas derivadas de la urbanización, y han fomentado la protección de la flora, la fauna y la biodiversidad, no han impedido la paulatina desaparición de las actividades agrarias tradicionales en las que se sustenta buena parte de su paisaje.

El proceso de inscripción de la Serra de Tramuntana en la lista de patrimonio Mundial recogió la necesidad de impulsar la conservación de la Serra como sistema agrario tradicional y único, y se incluyó como una de las acciones a desarrollar en el marco de la política de paisaje del Consell de Mallorca. La Estrategia de Paisaje de Mallorca (Dubon, 2009) se diseñó como una propuesta de aplicación del Convenio Europeo del Paisaje a la escala de la isla de Mallorca, utilizando los instrumentos al alcance de una institución como el Consell de Mallorca. De este modo, la propuesta de inscripción de la Serra de Tramuntana en la lista de Patrimonio Mundial tuvo la visión de unir, en un único proyecto, la visión de paisaje que plantea el Convenio de Florencia, y la del Centro de Patrimonio Mundial de UNESCO.

El éxito en el proceso de inscripción de la Serra de Tramuntana en la lista de Patrimonio Mundial pone las bases para establecer una política activa de conservación, en la línea que propone UNESCO para los paisajes culturales (Mitchell, 2009); sustentada en la implicación de la ciudadanía y en la protección de la adecuada interacción entre sociedad y medio ambiente en la que se fundamenta el concepto de paisaje cultural. La creación de figuras adecuadas y la gobernanza del territorio a través de un proyecto común debe permitir avanzar en la sensibilización social y en la salvaguarda de los valores de todo el territorio en su conjunto, y a la vez evitar los riesgos que, en relación al atractivo turístico y a la masificación, podría comportar la implantación de una marca como la de Patrimonio Mundial.

\section{BIBLIOGRAFÍA}

AAVV. (1998): Pla territorial parcial de la Serra de Tramuntana: fase d'informació $i$ diagnòstic. Conselleria d'Obres Públiques i Ordenació del Territori y Universitat de les Illes Balears. Palma.

BARCELÓ, M.; CARBONERO, M.A.; MARTÍ, R. y ROSSELLÓ-BORDOY, G. (1986): Les aigües cercades. Els qanât(s) de l'illa de Mallorca. Palma, Institut d'Estudis Baleàrics.

BAUZÀ, A. (2009): «El Aeropuerto de Palma de Mallorca. La puerta que abre el proceso de compresión espacio-temporal de la isla», en Ciudades, culturas y fronteras en un mundo en cambio. IX Coloquio de Geografía Urbana (Caravaca, I.; Fernández, V.,; y Silva, R., dir.). Sevilla, Consejería de Obras Públicas y Transportes, Junta de Andalucía, 259-276.

BINIMELIS, J.; GINARD, A. y SEGUÍ, J. (1999): «Le tourisme rural dans la dernière etape du nouveau modèle territorial de l'île de Majorque». En Insula. International Journal of Island Affairs núm. especial, pp. 61-64.

CAÑELLAS, N. (1997): El paisatge de l'Arxiduc. Institut d'Estudis Baleàrics. Palma. 
CARBONERO, M.A. (1984): «Terrasses per al cultiu irrigat i distribució social de l'aigua a Banyalbufar (Mallorca)». Documents d'Anàlisi Geogràfica, núm. 4, pp. 31-68.

COSGROVE, D. (2002): «Observando la naturaleza: el paisaje y el sentido europeo de la vista». Boletín de la Asociación de Geógrafos Españoles, nº 34, 63-89.

DUBON, M. (Dir.) (2009): Bases per a una estratègia de paisatge de Mallorca. Desenvolupament del Conveni Europeu del Paisatge. Fonaments, criteris, objectius $i$ línies d'acció. Palma, Consell de Mallorca - Departament de Territori.

DUBON, M. (Dir.) (2010): Cultural Landscape of the Serra de Tramuntana. Proposal for inscription in the World Heritage List (UNESCO). Island of Mallorca, Spain. Expediente de candidatura para la inscripción de la Serra de Tramuntana en la lista de Patrimonio Mundial (inédito). Disponible en http://whc.unesco.org/uploads/nominations/1371.pdf y http://www.serradetramuntana.net/ca/medialist/documentacio/ (consultada en agosto de 2012).

FOWLER, P. (2002): «World Heritage Cultural Landscapes, 1992-2002: a Review and Prospect», Cultural Landscapes: the Challenges of Conservation. Col. World Heritage Papers, $\mathrm{n}^{\mathrm{o}}$ 7. Paris, UNESCO World Heritage Centre.

GARCÍA, A.; DELGADO, B. y OJEDA, J. (2007): «Paisajes simbólicos de la ciudad de Sevilla». Ería, no 73-74, pp. 291-310.

GRIMALT, M. y BLÁZQUEZ. M: (1989): «El mapa de marjades de la Serra de Tramuntana». Treballs de Geografía no .42 pp. 43-57.

GRIMALT, M.; RODRÍGUEZ, R.; FERRER, I.; REYNÉS, A. y ALOMAR, G. (2002): «Paisatge i pedra en sec a Mallorca». Mètode, no 36, pp. 40-43.

GORMSEN, E. (1997): «The impact of tourism on coastal areas». Geojournal, n 42(1), pp 39-54.

KIRCHNER, H. (1994): «Espais irrigats andalusins a la Serra de Tramuntana i la seva vinculació amb el poblament». En Afers: fulls de recerca i pensament, Vol. 9, nº18, pp. 313-336.

KIRCHNER, H y SOTO, R. (2006): «Les tècniques agràries andalusines i les transformacions posteriors a la conquesta catalana a les Illes Balears». En Història de la Ciència a les Illes Balears. Vol. 1 (Bonner, A. y Bujosa, F., dir), 101-152. Palma, ed. Lleonard Muntaner.

MITCHELL, N.; RÖSSLER, M. y TRICAUD, P.M. (2009): World Heritage Cultural Landscapes. A Handbook for Conservation and Management. Col. World Heritage Papers, $\mathrm{n}^{\circ}$ 26. París, Centro de Patrimonio Mundial. UNESCO.

NOGUÉ, J. (Ed.) (2007): La construcción social del paisaje. Col. Paisaje y Teoría, nº 1 Madrid, Biblioteca Nueva.

NOGUÉ, J. (Ed.) (2008): El paisaje en la cultura contemporánea. Col. Paisaje y Teoría, n4. Madrid, Biblioteca Nueva.

PUJOL, M. (2000): «La Serra de Tramuntana: un territori de la identitat mallorquina. L'abast d'un parc natural». Bolletí de Geografia Aplicada (BdGA), vol. 2, pp. 21-26. Palma, Associació de Geògrafs de les Illes Balears.

RAMON, D. (2002): «Natura», en Estudi Ambiental, Cultural $i$ de Desenvolupament Sostenible de la Serra de Tramuntana (Equip SerraT). Palma, Conselleria de Medi Ambient. Govern de les Illes Balears. 
RIERA, M. y SOBERATS, N. (1991): «El sistema hidràulic d'Alaró (Mallorca)». En Bolletí de la Societat Arqueológica Lul.liana, nº47, pp 61-73.

REYNÉS, A. (2000): La construcció de pedra en sec a Mallorca (2a ed.). Palma, FODESMA - Consell de Mallorca. Disponible en http://www.conselldemallorca.net/media/15171/ pedraensec.pdf

ROBERTSON, I y RICHARDS, P. (Eds.) (2003): Studying cultural landscapes. Londres, Arnold / Nueva York, Oxford University Press.

ROGER, A. (1997): Court traité du paysage. París, Gallimard. Bibliothèque des Sciences Humaines.

ROSSELLÓ, V.M. (1964): «El regadío en la isla de Mallorca», en Aportación española al XX Congreso Internacional de Geografía. Madrid, Centro Superior de Investigaciones Científicas, pp. 235-254.

RÖSSLER, M. (2006): «World Heritage cultural landscapes: a UNESCO flagship programme 1992 - 1996». Landscape Research, vol. 31, núm. 4, 333-353.

ROUDIÉ, P. (2002): «El Paisaje y los Parajes del Patrimonio Mundial de la Humanidad de la UNESCO». En Paisaje y ordenación del territorio (Zoido Naranjo, F.-Venegas Moreno, C., Coord.). Sevilla, Junta de Andalucía-Fundación Duques de Soria, 92-99.

RULLÁN, O. (2004): «Una aproximació a la geografia històrica de Mallorca». En Treballs de la Societat Catalana de Geografia, no 57, pp. 85-109. Disponible en http://revistes.iec. cat/index.php/TSCG/article/view/54651/54847 (consultada en agosto 2012).

SABATÉ, J. (2008): «Paisajes culturales y proyecto territorial» en El paisaje en la cultura contemporánea (J. Nogué, Ed.), col. Paisaje y Teoría, Madrid, Biblioteca Nueva.

SALVÀ, P. (1998): «La Serra de Tramuntana: la transició de l'etapa preturística a l'actualitat». En Serra de Tramuntana: aportacions per a un debat (Rodriguez Perea, Coord.). Palma. Sa Nostra, Obra Social i Cultural.

SAUER, C. (1925): The Morphology of Landscape, Berkeley, University of California Publications in Geography, vol. 2, pp. 19-54.

SILVA, R. (2009): «Agricultura, paisaje y patrimonio territorial. Los paisajes de la agricultura vistos como patrimonio». Boletín de la Asociación de Geógrafos Españoles, n 49, pp. 309-334.

UNESCO (2006): Textos básicos de la Convención de Patrimonio Mundial de 1972. París, Centro del Patrimonio Mundial de la UNESCO.

VON DROSTE, B.; RÖSSLER, M. y TITCHEN, S. (Eds.) (1999): Linking Nature and Culture. Report on the Global Strategy. Natural and Cultural Heritage Expert Meeting, 25 to 29 March 1998, Amsterdam, Netherlands. París, UNESCO. 